\title{
USO DE CAE COMO FERRAMENTA DE ENSINO - APRENDIZAGEM NA DISCIPLINA DE MECANISMOS
}

Luciana Lima Monteiro - lucianamonteiro@ recife.ifpe.edu.br Instituto Federal de educação, ciência e tecnologia de Pernambuco Av. Prof. Luís Freire, 500 - Cidade Universitária 50740-545 - Recife - PE

José A. Peixoto da Costa - angelocosta@ recife.ifpe.edu.br Instituto Federal de educação, ciência e tecnologia de Pernambuco

Daniel C.dos Santos Pessoa - danielcalebesp@gmail.com

Instituto Federal de educação, ciência e tecnologia de Pernambuco

Luzitano H. Costa Silva de Paula - luzitano_hugo@ hotmail.com Instituto Federal de educação, ciência e tecnologia de Pernambuco

Resumo: Um dos grandes desafios do ensino na engenharia é motivar os estudantes principalmente nos períodos iniciais do curso, através da aplicação da teoria na prática, tornando os componentes curriculares mais dinâmicos e apresentando aos estudantes ferramentas modernas para resoluções de problemas reais de engenharia. Face à esta necessidade, este trabalho tem como objetivo apresentar uma análise numérica de um mecanismo de quatro barras através de uma ferramenta CAE (Computer Aided Engineering Engenharia Assistida por Computador), na disciplina de Mecanismos do Curso de Engenharia Mecânica. O mecanismo de quatro barras tem ampla aplicação em máquinas $e$ equipamentos e é de fundamental importância que os estudantes entendam o tipo de movimento gerado e a trajetória das barras que o compõem. Esta ferramenta vem auxiliar este entendimento através da simulação dos movimentos das barras, além de verificar o desempenho do mecanismo de quatro barras a partir de sua posição e velocidade. Desta forma, demonstrar que a utilização de simuladores é benéfica, a partir de conhecimentos dos processos analíticos. Após a utilização da ferramenta os alunos demonstraram melhor entendimento da disciplina e isto foi verificado através de relatórios onde os resultados foram avaliados por uma banca de professores.

Palavras-chave: Mecanismos de quatro barras. CAE. Ensino-aprendizagem. 


\section{INTRODUÇÃO}

Segundo Cordeiro, J. S. o desafio que o Brasil enfrenta na área das engenharias é tanto quantitativo como qualitativo. Do ponto de vista qualitativo, é preciso fortalecer a integração do sistema educacional com o sistema empresarial visando as necessidades da sociedade, das empresas, e no desenvolvimento tecnológico e econômico do país sendo de fundamental importância desenvolver novas metodologias para inserir o estudante na área de engenharia. Dentro deste contexto, uma das formas de integrar o estudante de engenharia é apresentá-lo recursos tecnológicos através de ferramentas de resoluções de problemas utilizados na indústria.

No curso de engenharia mecânica o componente curricular Mecanismos é ministrado geralmente no $5^{\circ}$ período. Esse componente faz parte da subárea de projetos de máquinas e envolve cálculos analíticos, análises gráficas e análises cinemáticas de dispositivos e partes de máquinas. Conforme Mabie, H. H., o estudo de mecanismos pode ser definido como a parte de projeto de máquinas relacionado com o projeto cinemático de sistemas articulados, cames, engrenagens e trens de engrenagens. $\mathrm{Na}$ ementa da disciplina estuda-se a análise cinemática de mecanismos de quatro barras. Segundo Norton, R. L., o mecanismo de quatro barras é o dispositivo mais comum e usual em mecanismos. Ele é também extremamente versátil em termos de tipos de movimento que pode gerar. Além disso, o mecanismo de quatro barras deve estar entre as primeiras soluções para os problemas de controle de movimento a serem investigadas.

Na Figura 1 abaixo, é apresentado o mecanismo de quatro barras. As barras são denominadas de elos. Os elos são representados por números, sendo o elo 1 o elo terra que é a estrutura do mecanismo definida como qualquer elo fixo (sem movimento) com relação ao sistema de referência. $\mathrm{O}$ elo 2 é denominado de manivela que faz uma revolução completa ou oscila e é articulado à estrutura. $\mathrm{O}$ elo 4 tem rotação oscilatória e é articulado à estrutura. $\mathrm{O}$ elo 3 é denominado de acoplador ou biela, possui movimento complexo e não é articulado à estrutura.

Figura 1- Mecanismo de quatro barras.

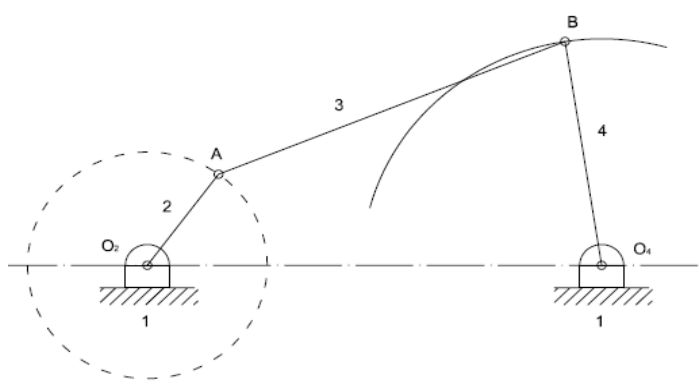

Fonte: Mabie, H. H.

O mecanismo de quatro barras é extensivamente utilizado na indústria como pode-se verificar em publicações como a de Baykus, B., que descreve um projeto de um mecanismo para abertura e fechamento de uma porta de bagageiro de ônibus, no qual foi feito uma analogia com um mecanismo de quatro barras para a análise cinemática do projeto. $\mathrm{Na}$ área de 
engenharia biomédica, Farhat, N., relata a determinação de forças no joelho humano usando um mecanismo de quatro barras. De forma geral, o estudo de Mecanismos é utilizado quando se deseja determinar a precisão da trajetória de pontos de partes de máquinas.

$\mathrm{Na}$ síntese gráfica de mecanismos pode-se utilizar ferramentas tradicionais como régua, par de esquadros e transferidor. Mas com estas ferramentas, se gasta muito tempo para obter a trajetória completa do mecanismo e o resultado não é preciso. Segundo Pivetta, C. S. et al as velocidades e as acelerações são importantes no resultado do funcionamento do mecanismo e a determinação pode ser feita por procedimentos gráficos, mas não permitem alta precisão nos resultados e destinam-se a poucos pontos de interesse. Ao se utilizar os procedimentos gráficos para a análise cinemática empregando sistemas auxiliares de desenho, os erros podem ser minimizados bem como o respectivo tempo de análise. As ferramentas como CAD (Computer-aided Drafting - Projeto assistido por computador) podem aumentar a velocidade deste processo além de melhorar a precisão dos resultados.

A partir de então vem se desenvolvendo metodologias de ensino para melhorar a entendimento do estudante em relação a trajetória do movimento dos mecanismos e a visualização instantânea do funcionamento dos mecanismos, ou seja, facilitar o entendimento através da utilização dos softwares, desta forma tornar a disciplina mais interativa e os estudantes motivados para aprender.

O Norton, R. L., utiliza um software específico para ensino de mecanismos, é completo do ponto de vista numérico e com boa visualização gráfica e limitação de interação. Silva, J. C. S. et al apresentam simulações de alguns mecanismos no ambiente MATLAB. Shiino, E. $\mathrm{T}$, apresenta uma ferramenta genérica, de síntese, análise e otimização de um mecanismo de quatro barras através do ambiente MATLAB. Souza, A. D. et al utilizam o MS Excel para o projeto de um mecanismo de quatro barras para uma trajetória definida. González, D. et al apresentam um aplicativo Android para o estudo cinemático do mecanismo plano de quatro barras.

O CAE (Computer Aided Engineering - Engenharia assistida por computador), é uma ferramenta utilizada para o desenvolvimento de projetos de engenharia através da simulação computacional. A simulação computacional é amplamente utilizada para realizar análises e melhorar a qualidade dos produtos e projetos. Com a interação dos softwares CAD com a ferramenta CAE permite alcançar melhores resultados com análises mais eficientes e ágeis, afirma Mirlisenna, G.

Diante do exposto acima, este trabalho apresenta uma análise númerica de um exercício do livro texto da disciplina de Mecanismos do curso de Engenharia mecânica através da ferramenta CAE. Foi determinado o deslocamento máximo, a velocidade e a força da junta do seguidor de um mecanismo de quatro barras. Espera-se com este trabalho contribuir para o aperfeiçoamento do ensino da disciplina de mecanismos para assim fortalecer a integração do sistema educacional com o sistema empresarial para formar hoje o engenheiro do amanhã.

\section{PROBLEMÁTICA}

$\mathrm{Na}$ forma tradicional de lecionar a disciplina de mecanismos os exercícios são resolvidos de forma analítica. A síntese analítica é uma técnica para resolver a análise cinemática. A álgebra utilizada torna a resolução mais fácil de ser resolvida por meios computacionais. Por outro lado, a visualização do movimento do mecanismo apenas pelos cálculos dificulta o entendimento do aluno. Dessa forma, a ferramenta $C A E$, resolve os cálculos, analisa os resultados e simula o movimento do mecanismo. A seguir são apresentadas as equações para a análise de posição e velocidade de um mecanismo de quatro barras. 


\subsection{Análise de posição do mecanismo de quatro barras}

Para definir a posição de todos os elos de um mecanismo de quatro barras é necessário o conhecimento de um parâmetro, o mais usual é o ângulo de entrada $\theta_{2}$ e os comprimentos dos elos, nomeados como a, b, c e d. É preciso determinar os ângulos $\theta_{3}$ e $\theta_{4}$, que é a posição da barra 3 denominada de acoplador e da barra 4 denominado de seguidor respectivamente. $\mathrm{Na}$ Figura 2 abaixo está apresentado o mecanismo de quatro barras com os ângulos de posição das barras.

Figura 2- Mecanismo de quatro barras com os ângulos de posição.

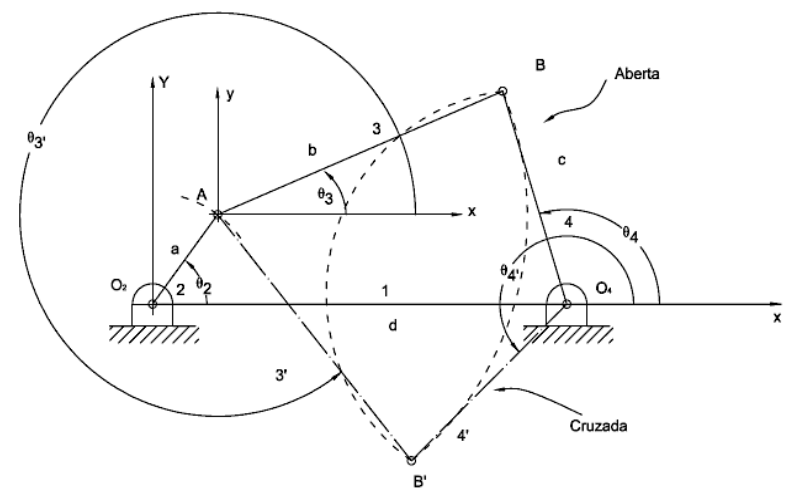

Fonte: Adaptado do Norton (2010).

Uma maneira de desenvolver e derivar as equações para posição, velocidade e aceleração dos mecanismos é considerar cada barra como um vetor e usar a notação de números complexos neles. Será adotado o método descrito no Norton, R. L. As barras são definidas como vetores:

$$
R_{2}+R_{3}-R_{4}-R_{1}=0
$$

Em seguida será substituída a notação de número complexo para cada vetor de posição e os comprimentos das quatros barras designadas como $a, b, c$ e $d$. A equação torna-se:

$$
a e^{j \theta_{2}}+b e^{j \theta_{3}}-c e^{j \theta_{4}}-d e^{j \theta_{1}}=0
$$

As expressões algébricas que definem $\theta_{3}$ e $\theta_{4}$ tem a seguinte forma:

$$
\begin{aligned}
& \theta_{3}=f\left\{a, b, c, d, \theta_{2}\right\} \\
& \theta_{4}=g\left\{a, b, c, d, \theta_{2}\right\}
\end{aligned}
$$

Então temos a seguinte equação:

$$
\begin{gathered}
a\left(\cos \theta_{2}+j \sin \theta_{2}\right)+b\left(\cos \theta_{3}+j \sin \theta_{3}\right)-c\left(\cos \theta_{4}+j \sin \theta_{4}\right) \\
-d\left(\cos \theta_{1}+j \sin \theta_{1}\right)=0
\end{gathered}
$$

Resolvendo a equação acima, temos: 
$b^{2}=a^{2}+c^{2}+d^{2}-2 a d \cos \theta_{2}+2 c d \cos \theta_{4}-2 a c\left(\sin \theta_{2} \sin \theta_{4}+\cos \theta_{2} \cos \theta_{4}\right)$

Para simplificar a expressão acima, as constantes $K_{1}, K_{2}$ e $K_{3}$ são definidas em termos dos comprimentos das barras:

$$
K_{1}=\frac{d}{a} \quad K_{2}=\frac{d}{c} \quad K_{3}=\frac{a^{2}-b^{2}+c^{2}+d^{2}}{2 a c}
$$

O ângulo de entrada $\theta_{2}$ e os comprimentos das barras serão agrupados como as constantes $A, B$ e $C$ :

$$
\operatorname{Atan}^{2}\left(\frac{\theta_{4}}{2}\right)+B \tan \left(\frac{\theta_{4}}{2}\right)+C=0
$$

Em que:

$$
\begin{gathered}
A=\cos \theta_{2}-K_{1}-K_{2} \cos \theta_{2}+K_{3} \\
B=-2 \sin \theta_{2} \\
C=K_{1}-\left(K_{2}+1\right) \cos \theta_{2}+K_{3}
\end{gathered}
$$

A solução da Equação (7) será:

$$
\theta_{4}=2 \arctan \left(\frac{-B \pm \sqrt{B^{2}-4 A C}}{2 A}\right)
$$

Para determinar $\theta_{3}$ apartir da Equação (4), isolando $\theta_{4}$, temos:

$$
\begin{gathered}
c \cos \theta_{4}=a \cos \theta_{2}+b \cos \theta_{3}-d \\
c \sin \theta_{4}=a \sin \theta_{2}+b \sin \theta_{3}
\end{gathered}
$$

Resolvendo a Equação (10) acima para $\theta_{3}$ temos:

$$
K_{1} \cos \theta_{3}+K_{4} \cos \theta_{2}+K_{5}=\cos \theta_{2} \cos \theta_{3}+\sin \theta_{2} \sin \theta_{3}
$$

A constante $K_{l}$ é a mesma da Equação (6) e $K_{4}$ e $K_{5}$ são:

$$
K_{4}=\frac{d}{b} ; \quad K_{5}=\frac{c^{2}-d^{2}-a^{2}-b^{2}}{2 a b}
$$

Então será obtida a forma seguinte:

$$
\operatorname{Dtan}^{2}\left(\frac{\theta_{3}}{2}\right)+\operatorname{Etan}\left(\frac{\theta_{3}}{2}\right)+F=0
$$

Em que:

$$
\begin{gathered}
D=\cos \theta_{2}-K_{1}+K_{4} \cos \theta_{2}+K_{5} \\
E=-2 \sin \theta_{2} \\
F=K_{1}+\left(K_{4}-1\right) \cos \theta_{2}+K_{5}
\end{gathered}
$$


C COBENCE 2020

"Os desafios para formar hoje o engenheiro do amanhã"
$\mathrm{Ol}$ a $\mathrm{O} 3$ de dezembro Evento On-line

A solução da Equação (14) acima é:

$$
\theta_{3}=2 \arctan \left(\frac{-E \pm \sqrt{E^{2}-4 D F}}{2 D}\right)
$$

A partir das Equações (9) e (15) são definidas as posições dos elos 3 e 4 respectivamente.

\subsection{Análise de velocidade do mecanismo de quatro barras}

No mecanismo de quatro barras a determinação da velocidade é necessária para o cálculo da energia cinética armazenada e para determinar as acelerações das barras, as quais são necessárias para o cálculo das forças dinâmicas. Para encontrar a equação da velocidade deriva-se a Equação (2) em relação ao tempo:

$$
j a e^{j \theta_{2}} \frac{d \theta_{2}}{d t}+j b e^{j \theta_{3}} \frac{d \theta_{3}}{d t}-j c e^{j \theta_{4}} \frac{d \theta_{4}}{d t}=0
$$

Mas,

$$
\frac{d \theta_{2}}{d t}=\omega_{2} ; \quad \frac{d \theta_{3}}{d t}=\omega_{3} ; \quad \frac{d \theta_{4}}{d t}=\omega_{4}
$$
abaixo:

A partir da Equação (17), encontra-se as velocidades angulares dos elos 3 e 4 da forma

$$
\omega_{3}=f\left(a, b, b, b, \theta_{2}, \theta_{3}, \theta_{4}, \omega_{2}\right) \quad \omega_{4}=g\left(a, b, c, d, \theta_{2}, \theta_{3}, \theta_{4}, \omega_{2}\right)
$$

Resolvendo a Equação (17), é determinada a Equação das velocidades angulares dos elos 3 e 4 do mecanismo de quatro barras:

$$
\begin{gathered}
\omega_{3}=\frac{a \omega_{2} \sin \left(\theta_{4}-\theta_{2}\right)}{b \sin \left(\theta_{3}-\theta_{4}\right)} \\
\omega_{4}=\frac{a \omega_{2} \sin \left(\theta_{2}-\theta_{3}\right)}{c \sin \left(\theta_{4}-\theta_{3}\right)}
\end{gathered}
$$

Para as equações das velocidades lineares é necessário substituir as identidades de Euler:

$$
\begin{aligned}
& V_{A}=j a \omega_{2}\left(\cos \theta_{2}+j \sin \theta_{2}\right)=a \omega_{2}\left(-\sin \theta_{2}+j \cos \theta_{2}\right) \\
& V_{B A}=j b \omega_{3}\left(\cos \theta_{3}+j \sin \theta_{3}\right)=b \omega_{3}\left(-\sin \theta_{3}+j \cos \theta_{3}\right) \\
& V_{B}=j c \omega_{4}\left(\cos \theta_{4}+j \sin \theta_{4}\right)=c \omega_{4}\left(-\sin \theta_{4}+j \cos \theta_{4}\right)
\end{aligned}
$$

Os termos reais e imaginários são os componentes $x$ e $y$, respectivamente. As Equações (19), (20), (21), (22) e (23) acima fornecem uma solução completa para as velocidades 


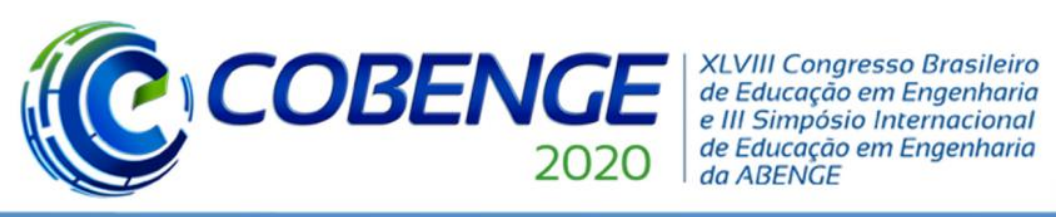

"Os desafios para formar hoje o engenheiro do amanhã"

angulares dos elos e das velocidades das juntas em um mecanismo de quatro barras com junta pinada. As equações acima são utilizadas na metodologia tradicional do ensino da disciplina de mecanismos.

\section{METODOLOGIA}

O desenvolvimento da metodologia deste trabalho é baseado na resolução de um exercício do livro texto da disciplina de Mecanismos, o Norton, R. L. Inicialmente foi feito pelo estudante uma análise de posição e velocidade através da síntese analítica utilizando as Equações (9) e (23) respectivamente. Após esta etapa, os alunos resolveram o mesmo exercício utilizando a ferramenta $C A E$ da Ansys. Esta ferramenta tem como base a análise númerica por elementos finitos.

O mecanismo é não Grashoff de quatro barras saída no acoplador de duas posições com deslocamento complexo (geração de movimento). Sendo determinado o deslocamento máximo do acoplador, velocidade do elo 4 e a força na junta do acoplador. Na Figura 3 abaixo está apresentado o mecanismo projetado através do Ansys. Este mecanismo gera um movimento complexo alternativo no acoplador.

Figura 3- Mecanismo com saída do acoplador possuindo duas posições e movimento complexo.

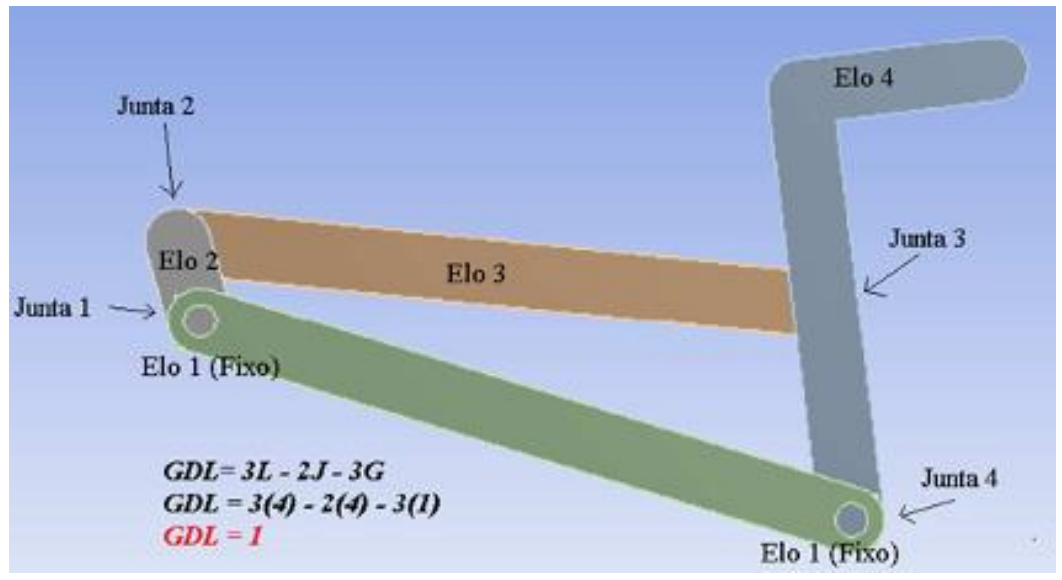

Fonte: Ferramenta Ansys (2019).

\section{RESULTADOS}

As Figuras de 4, 5 e 6 apresentam os resultados do deslocamento máximo, da velocidade e da força na junta do mecanismo, dada uma velocidade de entrada constante de $10 \mathrm{rad} / \mathrm{s}$ no elo 2. Observa-se que o deslocamento máximo do acoplador é de $0,0184 \mathrm{~m}$, a velocidade máxima é de $0,171 \mathrm{~m} / \mathrm{s}$ ao longo do acoplador e a força máxima na junta é de $3,6810^{-2} \mathrm{~N}$. 
Figura 4. Resultado obtido via simulação numérica do deslocamento.

\begin{tabular}{|l|l|}
\hline 0,018419 \\
0,016373 \\
0,014326 \\
0,012279 \\
0,010233 \\
0,0081863 \\
0,0061397 \\
0,0040931 \\
0,0020466 \\
0 Min
\end{tabular}

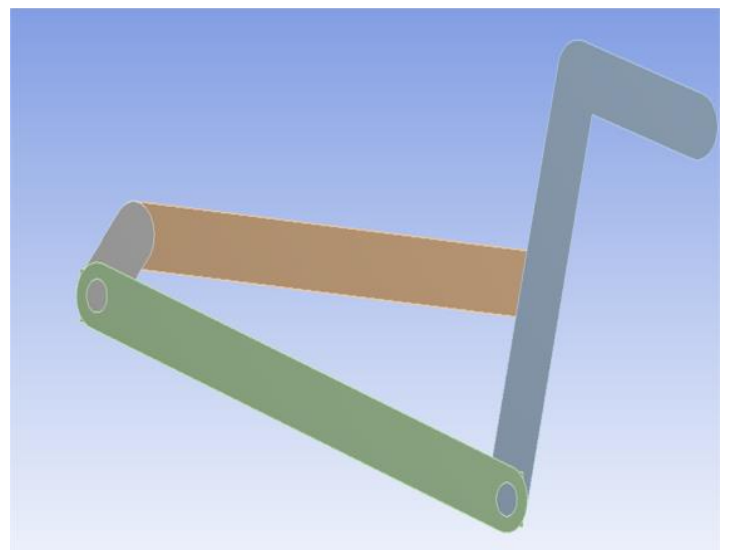

Fonte: ferramenta ansys (2019).

Figura 5. Resultado obtido via simulação numérica da velocidade.

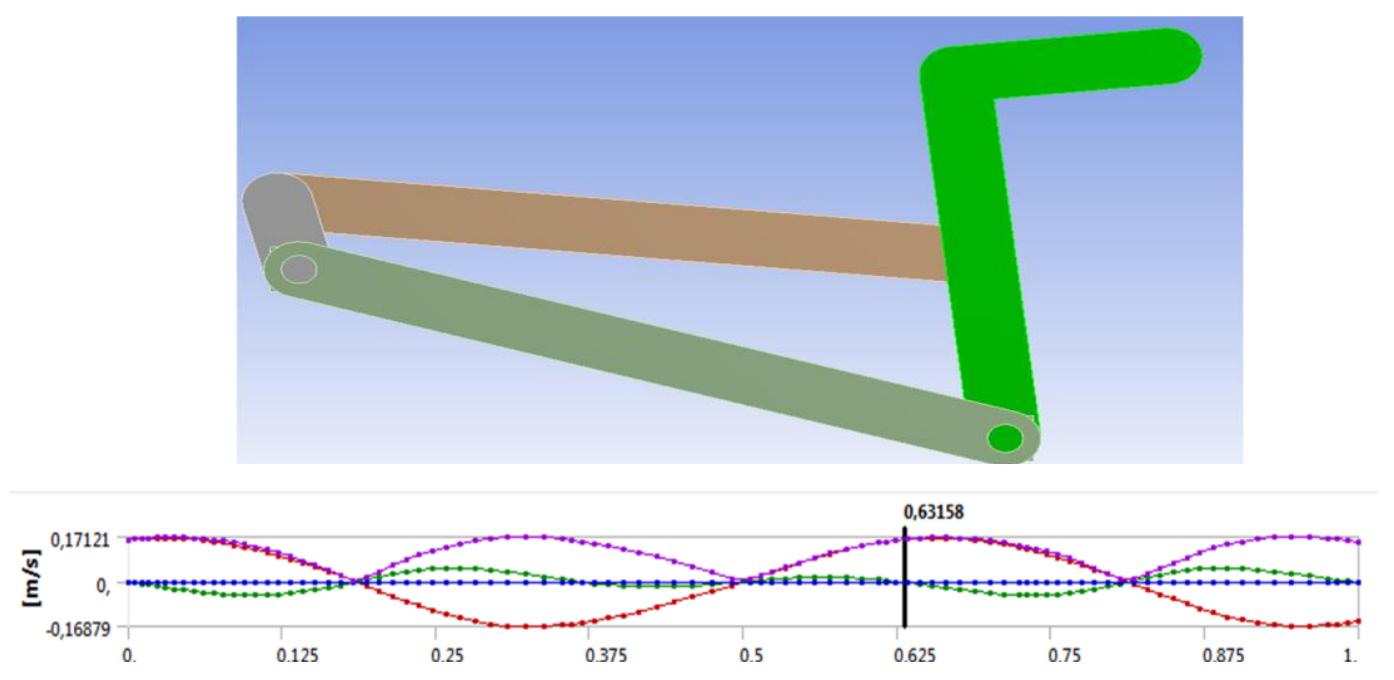

Fonte: ferramenta ansys (2019).

Figura 6. Resultado obtido via simulação numérica da força na junta
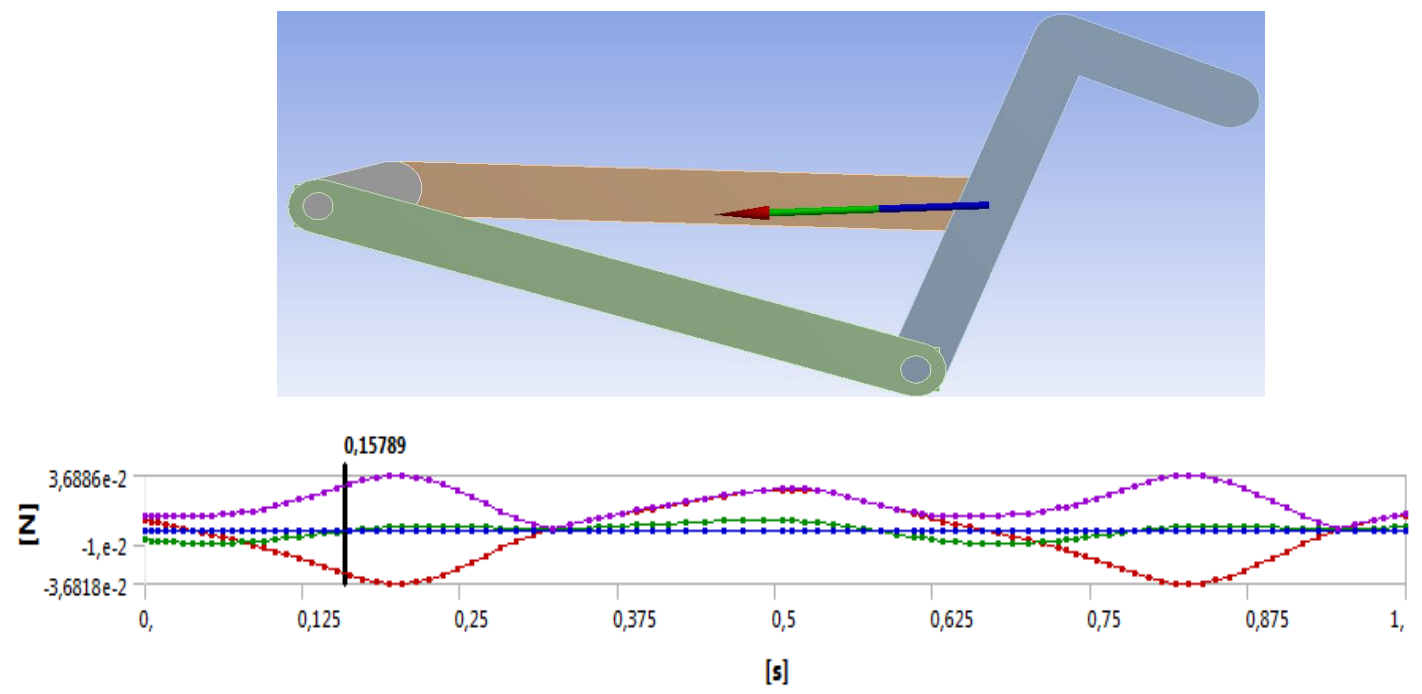

Fonte: ferramenta ansys (2019). 


\section{CONCLUSÃO}

Os resultados deste trabalho demostraram que os procedimentos propostos, ao se utilizar recursos computacionais permitem analisar a cinemática dos mecanismos estudados em todo seu ciclo. E podendo ser utilizado como base para outros sistemas mecânicos mais complexos. O relato dos estudantes sobre a experiência foi que este estudo possibilitou a eficiência do entendimento da disciplina de Mecanismos de maneira prática através da ferramenta CAE (Computer Aided Engineering - Engenharia Assistida por Computador). Ao final do semestre, os estudantes demostraram pleno entendimento dos principais conceitos da disciplina de Mecanismos. E desta forma consolidando a importância de apresentar ao estudante uma ferramenta de alto nível utilizado na indústria mundial na área de modelamento, simulação e análise de sistemas mecânicos.

\section{REFERÊNCIAS}

Ansys. Disponível em: https://www.ansys.com. Acesso em: 25/06/2019.

Baykus, B.; Anli, E.; Ozkol, I. Design and Kinematics Analysis of a Parallel Mechanism to be Utilized as a Luggage Door by an Analogy to a Four-Bar Mechanism, Engineering, p. 411421, 2011.

CORDEIRO, J. S.; ALMEIDA, N. N.; BORGES, M. N.; DUTRA, S. C.; VALINOTE, O. L.; PRAVIS, Z. M. C. Um futuro para a educação em engenharia no Brasil: desafios e oportunidades. Revista de Ensino de Engenharia, v27, n.3, p. 69-82, 2008.

FARHAT, N.; MATA, V.; ROSA, D.; FAYOS J. A procedure for estimating the relevant forces in the human knee using a four-bar mechanism, Journal Computer Methods in Biomechanics and Biomedical Engineering, vol13, n.5, p. 577-587, 2010.

GONZÁLEZ, D.; ESTRADA, E.; ROLDÁN, Y. J. Aplicacion android para el estúdio de mecanismo planos de cuatro barras. Entre Ciencia e Ingeniería, Barranquila, v.10, n.20, p. $9-25,2016$.

MABIE, H. H.; OCVIRK, F. W. Mechanisms and Dynamics of Machinery. New York: John Wiley \& Sons. 1978.

MIRLISENNA, G. Método dos Elementos Finitos: o que é? Disponível em: https://www.esss.co/blog/metodo-dos-elementos-finitos-o-que-e/. Acesso em: 27/03/2020.

NORTON, Robert L. Cinemática e dinâmica dos mecanismos. New York: Mc Graw Hill, 2010 .

PIVETTA, C. S.; REZENDE, O. P.; CAMPOS, L. M.; GRECHI, R.; BRANDÃO, J. G. T.; Cinemática de um mecanismo articulado com deslizamento e aceleração de coriolis. In: XI Congresso Nacional de Engenharia Mecânica, Metalúrgica e Industrial, 2011, Porto Alegre. Anais. 2011.

SHIINO, E. T. Síntese e Análise de Mecanismo de quatro-barras. 2017. Tese (mestrado) Faculdade de Engenharia Mecânica - UNICAMP, São Paulo, 2017. 
SILVA, J. C. S.; IRMÃO, M. A. da S.; SILVA, A. A.; Modelagem e simulação computacional de mecanismos para o ensino de engenharia. In: XXXIV Congresso Brasileiro de Engenharia, 2006, Passo Fundo. Anais. Passo Fundo, 2006.

SOUZA, A. D. et al. Projeto de mecanismos de quatro barras para obter uma trajetória desejada com auxílio computacional. In: X Encontro Latino Americano de Pós-Graduação, 2010, São Paulo. Anais. São Paulo, 2010.

\title{
USE OF CAE AS A TEACHING AND LEARNING TOOL IN THE COURSE OF MECHANISMS
}

\begin{abstract}
One of the big challenges of teaching in engineering is to motivate students mainly in the first year of the graduate, through the application of theory in practice, making the course more atractive and presenting students with modern tools for solving real engineering problems. In view of this need, this work aims to determine the displacement, speed and force at the coupler output joint of a four-bar mechanism through a CAE tool (Computer Aided Engineering), in the Mechanisms course of the Mechanical Engineering graduate. The fourbar mechanism has wide application in machines and equipment and it is of fundamental importance that students understand the type of movement generated and the trajectory of the bars that compose it. This tool helps to understand this by simulating the movements of the bars, in addition to verifying the performance of the mechanisms of four bars from their position and speed. In this way, demonstrate that the use of simulation tools is beneficial, based on knowledge of the analytical processes. After using the tool, the students demonstrated a better understanding of the course and this was verified through reports were the results were evaluated by a panel of teachers.
\end{abstract}

Keywords: four bar mechanisms, CAE, teaching and learning. 